\title{
Analysis of microRNA expression profile by small RNA sequencing in Down syndrome fetuses
}

\author{
YONG XU ${ }^{1,3^{*}}$, WUXIAN LI $^{2 *}$, XUEYAN LIU ${ }^{4}$, HUALIN MA ${ }^{4}$, ZHIGUANG TU ${ }^{3}$ and YONG DAI ${ }^{3,4}$ \\ ${ }^{1}$ Clinical Medical Research Center, Pingshan People's Hospital, Shenzhen, Guangdong 518118; \\ ${ }^{2}$ Department of Clinical Laboratory, Chongqing Maternal and Child Health Care Hospital, Chongqing 400013; \\ ${ }^{3}$ Key Laboratory of Laboratory Medical Diagnostics of Education Ministry, Chongqing Medical University, \\ Chongqing 400016; ${ }^{4}$ Clinical Medical Research Center, the Second Clinical Medical College of Jinan University \\ (Shenzhen People's Hospital), Shenzhen, Guangdong 518020, P.R. China
}

Received June 19, 2013; Accepted August 22, 2013

DOI: $10.3892 / \mathrm{ijmm} .2013 .1499$

\begin{abstract}
Down syndrome (DS) is caused by trisomy of human chromosome 21 (Hsa21) and is associated with numerous deleterious phenotypes, including cognitive impairment, childhood leukemia and immune defects. Five Hsa21-derived microRNAs (i.e., hsa-miR-99a, let-7c, miR-125b-2, miR-155 and miR-802) are involved in variable phenotypes of DS. However, the changes involved in the genome-wide microRNA expression of DS fetuses under the influence of trisomy 21 have yet to be determined. To investigate the expression characteristic of microRNAs during the development of DS fetuses and identify whether another microRNA gene resides in the Hsa21, Illumina high-throughput sequencing technology was employed to comprehensively characterize the microRNA expression profiles of the DS and normal fetal cord blood mononuclear cells (CBMCs). In total, 149 of 395 identified microRNAs were significantly differentially expressed (fold change $>2.0$ and $\mathrm{P}<0.001)$ and 2 of 181 candidate novel microRNAs were identified as residing within the 'DS critical region' of human chromosome 21 (chr21q22.2-22.3). Additionally, 7 of 14 Hsa21-derived microRNAs were detected, although not all seven were overexpressed in DS CBMCs compared with the control. Gene ontology enrichment analyses revealed that a set of abnormally expressed microRNAs were involved in the regulation of transcription, gene expression, cellular biosynthetic process and nucleic acid metabolic process. Significantly, most of the mRNA targets in these categories were associated with immune modulation
\end{abstract}

Correspondence to: Dr Yong Dai, Shenzhen People's Hospital, 1017 North Road, Dongmen, Shenzhen, Guangdong 518020, P.R. China

E-mail: daiyong22@yahoo.com.cn

*Contributed equally

Key words: Down syndrome, microRNA, high-throughput nucleotide sequencing, cord blood (i.e., SOD1, MXD4, PBX1, BCLAF1 and FOXO1). Findings of the present study provided a considerable insight into understanding the expression characteristic of microRNAs in the DS fetal CBMCs. To the best of our knowledge, this is the first study to examine genome-wide microRNA expression profiles in the DS fetus. Differentially expressed microRNAs may be involved in hemopoietic abnormalities and the immune defects of DS fetuses and newborns.

\section{Introduction}

Down syndrome (DS) is caused by the occurrence of three copies of chromosome 21 and is associated with a number of deleterious phenotypes, including cognitive impairment, childhood leukemia and immune defects. These complicated and varied phenotypes were generally thought to result from the interaction between human chromosome 21 (Hsa21) genes with other disomic genes (1). Recent bioinformatics annotation has indicated that Hsa21 harbors $>500$ genes, including 14 microRNA encoding genes (i.e., hsa-miR-99a, let-7c, miR-125b, miR-155, miR-802, miR-3197, miR-3648, miR-3687, miR-4327, miR-4759, miR-4760, miR-3118-5, miR-3156-3 and miR-548x) (2). Moreover, in previous studies, 5 of 14 Hsa21-derived miRNAs (i.e., hsa-miR-99a, let-7c, miR-125b-2, miR-155 and miR-802) have been proven to be correlated with the complex and variable phenotypes of DS and be overexpressed in the heart, frontal cortex and hippocampus of DS fetuses (1,3-5). However, the changes involved in the genome-wide miRNA expression of DS fetuses under the influence of trisomy 21 have yet to be determined. A miRNA can potentially regulate a large number of protein-coding genes, while a target gene can also be regulated by multiple miRNAs (6). Thus, the molecular regulating mechanisms of the underlying progression of complex and variable phenotypes of DS should be examined to identify the genome-wide expression patterns of miRNAs.

MicroRNAs (miRNAs) are a class of endogenous, 18-25 nucleotides (nt) long, single-stranded RNAs that have emerged as key post-transcriptional regulators of gene expression and play a key role in various cell processes, such as cell proliferation, differentiation, apoptosis, embryonic 
development and tissue differentiation (2,7-9). miRNAs are transcribed from intra- and inter-genetic regions of the genome by RNA polymerase II (10). Following their processing, mature miRNAs are assembled into ribonucleoprotein complexes known as miRNA-induced silencing complexes (RISC). RISC subsequently inhibits gene expression by perfect complementary binding, for mRNA degradation, or imperfect binding at the 3'UTR region, to inhibit translation (11). Its abnormal expression has been proven to be involved in the occurrence and development of diseases (11-14).

Hybridization-based methodologies, such as microarray and PCR-based assays, have been used thus far to identify and profile the association between the Hsa21-derived miRNAs and the variable phenotypes of DS. Sethupathy et al (5) first used quantitative PCR (qPCR) to study the Hsa21-derived miR-155 in fibroblasts from monozygotic twins discordant for trisomy 21 (one twin was unaffected, whereas the other had trisomy 21) and confirmed miR-155 was overexpressed in the fibroblasts from the twin with DS. This result may be relevant to the observed lower blood pressure in individuals with DS. Subsequently, Kuhn et al (3) assessed the miRNA expression profiles of DS hippocampus specimens using microarrays and verified that five Hsa21-derived miRNAs were overexpressed in the DS brain. Moreover, they confirmed that miR-155 and miR-802 are associated with cognitive impairment in individuals with DS by regulating the methyl-CpG-binding protein (MeCP2) gene $(4,13)$.

Additionally, a number of studies indirectly determined that Hsa21-derived miRNAs may partially contribute to most of the observed DS phenotypes. For example, hsa-miR-125b and miR-99a are associated with pediatric AML (non-DS) (15), 10 of 89 predicted miR-155 target genes are involved in hematopoietic and myeloproliferative disorders (14) and hsa-miR-99a, let-7c and miR-125b may act as tumor suppressors that play an important role in the reduced incidence of solid tumors in DS individuals (16-18). Alternatively, previous studies $(1,15-18)$ mainly focused on Hsa21-derived miRNA expression in tissues of human DS subjects, while few studies have focused on determining the expression profiles of miRNAs isolated from human blood samples. Thus, it would be clinically relevant to investigate early gene regulatory mechanisms associated with hemopoietic abnormalities and the immune defects in DS patients. Furthermore, it is easier to obtain blood samples compared with tissue samples.

In the present study, in order to investigate the expression characteristic of miRNAs during the development of DS fetus and to identify whether another miRNA gene is located on the Hsa21 (in addition to the confirmed 14 Hsa21-derived miRNAs), Illumina high-throughput sequencing technology was employed to comprehensively characterize the miRNA expression profiles in the cord blood of DS and control fetuses. Compared to previous hybridization-based methods, high-throughput sequencing methods have significant advantages. First, they can provide a more integrated view of the miRNAs transcriptome and identify modest or even low abundant miRNAs that previous methods are not able to detect. Second, this method can successfully identify novel miRNAs via the direct observation and validation of the folding potential of the flanking genomic sequences (19). Using Illumina sequencing technology, 395 known miRNAs and 181 novel candidate miRNAs were identified in this study. Moreover, 149 known miRNAs were significantly differentially expressed and two novel miRNAs were located in the 'DS critical region' of chromosome 21 (chr21q22.2-22.3). The data obtained in this study provides considerable insight into understanding the expression characteristic of miRNAs in the DS fetal cord blood mononuclear cells (CBMCs). Differentially expressed miRNAs may be associated with the hemopoietic abnormalities and the immune defects of DS fetuses and newborns.

\section{Materials and methods}

Clinical samples and ethics statement. A total of six DS and six matched control fetal cord blood samples (18-22 weeks of gestation) were obtained from the Shenzhen People's Hospital, China. Three DS and three control cord blood samples were combined to form pooled DS and control cord blood samples, respectively, for small RNA library construction and Illumina sequencing. The remaining three DS and three control samples were used as the validation set to confirm the miRNA differential expression patterns by qPCR. The cord blood samples were obtained by puncture extraction with the help of a color Doppler ultrasound as the prenatal women had been undergoing prenatal diagnosis. The samples were then verified by protein electrophoresis to exclude the contamination of maternal blood cells. The characteristics of each case are provided in the Table I. Written informed consent for the biological studies was obtained from all the participants. The study was approved by the Ethics Committee of the Shenzhen People's Hospital.

CBMCs were separated by a Ficoll-Paque (Sigma, St. Louis, MO, USA) density gradient centrifugation according to the manufacturer's instructions. Briefly, $2 \mathrm{ml}$ of blood (with EDTA as an anticoagulant) was layered on $3 \mathrm{ml}$ of Ficoll-Hypaque (Sigma) and centrifuged for $25 \mathrm{~min}$ at $1,300 \mathrm{rpm}$ at room temperature. Mononuclear cells at the interface were aspirated with a Pasteur pipette, washed twice in PBS by centrifugation for $10 \mathrm{~min}$ at $900 \mathrm{rpm}$ at room temperature and dissolved in $1 \mathrm{ml}$ of TRIzol ${ }^{\circledR}$ reagent (Invitrogen, Carlsbad, CA, USA). The samples were then stored at $-80^{\circ} \mathrm{C}$ until further use.

Total RNA isolation and sequencing. Total RNA isolation was performed with CBMCs using TRIzol reagent (Invitrogen) according to the manufacturer's instructions. Small RNA library preparation and sequencing were performed with Illumina sequencing technology (BGI-Shenzhen, Shenzhen, China). Briefly, the small RNA (sRNA) population was isolated by separating $10 \mu \mathrm{g}$ of total RNA using denaturing polyacrylamide gel electrophoresis (PAGE) and excising the portion of the gel corresponding to the appropriate size (15-30 nt) based on standard oligonucleotide markers. The sRNA was ligated with 3' (5'-pUCGUAUGCCGUCUUCUGCUUGidT-3') and 5' (5'-GUUCAGAGUUCUACAGUCCGACGAUC-3') adapters using T4 RNA ligase and purified on a $15 \%$ Tris-Borate-EDTA (TBE) urea PAGE. The modified sRNA was then reverse transcribed by Illumina's small RNA RT-Primer (5'-CAAGCAG AAGACGCATACGA-3'). The cDNA was used as the template for PCR amplification (15 cycles) using Illumina's small RNA primer set (5'-CAAGCAGAAGACGGCATACGA-3' and 5'-AATGATACGGCGACCACCGACAGGTTCAGAGTTCT 
Table I. The characteristics of each case.

\begin{tabular}{|c|c|c|c|c|c|}
\hline \multirow[b]{2}{*}{ Mother-ID } & \multirow[b]{2}{*}{ Age (years) } & \multirow{2}{*}{$\begin{array}{l}\text { Gestational } \\
\text { age (weeks) }\end{array}$} & \multirow{2}{*}{$\begin{array}{c}\text { The result of } \\
\text { karyotype }\end{array}$} & \multicolumn{2}{|c|}{ Protein electrophoresis } \\
\hline & & & & $\operatorname{HBA}(\%)$ & $\operatorname{HBF}(\%)$ \\
\hline Patient $1^{\mathrm{a}}$ & 44 & 20 & $47, X X,+21$ & 5.5 & 94.5 \\
\hline Patient $2^{\mathrm{a}}$ & 36 & 20 & $47, X X,+21$ & 6.7 & 93.3 \\
\hline Patient $3^{a}$ & 30 & 21 & $47, X Y,+21$ & 4.8 & 95.2 \\
\hline Patient 4 & 34 & 20 & $47, \mathrm{XX},+21$ & 6.2 & 93.8 \\
\hline Patient 5 & 33 & 22 & $47, X Y,+21$ & 5.7 & 94.3 \\
\hline Patient 6 & 32 & 19 & $47, X X,+21$ & 6.4 & 93.6 \\
\hline Control $1^{\mathrm{a}}$ & 37 & 21 & $46, X X$ & 4.8 & 95.2 \\
\hline Control $2^{\mathrm{a}}$ & 36 & 20 & $46, X Y$ & 6.4 & 93.6 \\
\hline Control $3^{\mathrm{a}}$ & 30 & 20 & $46, X X$ & 5.8 & 94.2 \\
\hline Control 4 & 34 & 18 & 46,XY & 6.8 & 93.2 \\
\hline Control 5 & 33 & 22 & $46, X X$ & 6.6 & 93.4 \\
\hline Control 6 & 32 & 21 & $46, X X$ & 5.2 & 94.8 \\
\hline
\end{tabular}

${ }^{a}$ The cases were selected to perform high-throughput nucleotide sequencing and the remaining cases were used as the validation set. HBA, adult hemoglobin; HBF, fetal hemoglobin.

ACAGTCCGA-3'). The amplified cDNAs were then purified by $6 \%$ TBE PAGE and sequenced on the Illumina Hi-Seq 2000 according to the manufacturer's instructions.

Read filter and small RNA annotation. Two sRNA sequencing data sets comprising the DS and control CBMCs were obtained from Illumina fast track sequencing services and were submitted to the NCBI's Gene Expression Omnibus (http://www.ncbi.nlm.nih.gov/geo/) under series the accession number GSE39436. The low quality reads were filtered out to exclude those that were most likely to represent the sequencing errors and $3^{\prime} / 5^{\prime}$ adaptor sequences were subsequently trimmed to clean full-length reads and formatted into a non-redundant FASTQ format. The frequencies of each unique small RNA sequence reads were calculated as sequence tags (the number of reads for each sequence reflects its relative abundance) and only small RNA sequences ranging from 18 to $30 \mathrm{nt}$ were retained for subsequent analysis.

Unique sequencing reads that passed the filters were mapped onto the reference human genome using the SOAP (version 2.0) program with at most two mismatches (20). Subsequently, the unique sequence reads that mapped to the human genome were aligned against the miRBase version 17.0, Rfam 10.0 (http://rfam.sanger.ac.uk/), piRNABank (NCBI) and the human gene UCSC annotation hg19 (http://genome. ucsc.edu/). Overlapping annotation sequences from these data were classified as miRNA, tRNA, rRNA, mRNA, snoRNA, snRNA, piRNA or other non-coding RNAs. The unannotated sequences that did not overlap with any of these annotations but could be coded by intergenic and intronic regions of the human genome, served as a source of potential novel miRNAs (11).

Normalization of the calculation of transcript parts per million (TPM). A normalization step was performed because the total number of reads from different experiments was not the same and variations in the number of reads of individual miRNA were possibly due to sequencing depth. The number of reads from a unique sequence (representing a miRNA) was divided by the total clone count of the sample and multiplied by $10^{6}$. The total clone count was the sum of frequencies of all the residual unique sequences after filtering.

Expression analysis of known miRNAs and the prediction of novel miRNAs. Differentially expressed miRNAs between the two libraries were identified by the fold-change method, as described in a previous study (9). The statistical significance (P) was inferred based on the Bayesian method, which was developed for the analysis of digital gene expression profiles and can account for the sampling variability of tags with low counts (21). Unless stated otherwise, the unique sequences with >10 TPM in the two libraries was ignored and the most frequently observed isomiR was used as the diagnostic sequence for comparison of miRNA expression between libraries. A specific miRNA was deemed to be significantly differentially regulated if the $\mathrm{P}$ determined by this method was $\leq 0.001$ and there was at least a 2 -fold change in the normalized sequence counts.

The unannotated sequences that could be aligned to the human genome were considered for detecting novel candidate miRNAs. Briefly, the 100 nucleotides of genomic sequences flanking each side of the unannotated sequences were extracted. The RNA secondary structures with a folding free energy of less than or equal to $-18 \mathrm{kcal} / \mathrm{mol}$ (Mfe $\leq-18$ $\mathrm{kcal} / \mathrm{mol}$ ) were predicted using RNAfold (http://rna. tbi.univie. ac.at/cgi-bin/RNAfold.cgi). The secondary structures were analyzed by MIREAP (default setting) (http://sourceforge.net/ projects/mireap/), which is a sophisticated tool commonly used to identify novel miRNAs from high-throughput sequencing data and has been widely used in related studies $(22,23)$. Concomitantly, the folded structures identified were further tested by a publicly available miRNA classifier (miRDeep) 
to reduce the number of false positives (24). Additionally, the sRNAs derived from the same precursors were grouped into a common family and the most abundant member was selected as the representative of the family.

$q P C R$. qPCR was performed as described previously (3), with minor modifications. Briefly, total RNA was isolated from CBMCs using TRIzol reagent (Invitrogen) as per the manufacturer's instructions. The RNAs were reverse transcribed to cDNA with stem-loop-like RT primers (GenePharma, Shanghai, China) that are specific for only the mature miRNA species and then quantified on an Applied Biosystems 7500 Real-Time PCR system (Applied Biosystems, Carlsbad, CA, USA) using SYBR-Green PCR Master Mix (Toyobo, Osaka, Japan). The PCR reaction was conducted at $95^{\circ} \mathrm{C}$ for $5 \mathrm{~min}$, followed incubations at $95^{\circ} \mathrm{C}$ for $15 \mathrm{sec}, 65^{\circ} \mathrm{C}$ for $15 \mathrm{sec}$ and $72^{\circ} \mathrm{C}$ for $32 \mathrm{sec}$ and repeated for 40 cycles. Each PCR was repeated at least three times. The relative expression level of each miRNA was normalized against RNU6B expression levels. The fold-change was calculated according to the $2^{-\Delta \Delta C \mathrm{~T}}$ method.

Target prediction and gene ontology $(G O)$ analysis. As previously described (9), the target genes for each significantly differentially regulated miRNA were predicted by miRecords (http://mirecords.biolead.org/), which integrates the predicted targets from the following miRNA target prediction tools: DIANA-microT, MicroInspector, miRanda, MirTarget2, miTarget, NBmiRTar, PicTar, PITA, RNA22, RNAhybrid and TargetScan/TargertScanS. Since miRNA target prediction often suffers from a high false-positive rate, only the target genes supported by at least 5 of 11 established target prediction programs compiled by miRecords were taken into account.

The Gene Ontology (GO) terms of the predicted targeted genes were annotated using the DAVID gene annotation tool (http://david.abcc.ncifcrf.gov/summary.jsp). As previously described (25), the GeneMerge software was employed for identification of the significant overrepresentation of particular GO terms. GO terms and miRNAs were clustered using hierarchical clustering in $\mathrm{R}$. A heatmap was created in $\mathrm{R}$ using the heatmap function (default parameters).

\section{Results}

Sequencing and annotation of SRNA. Using Illumina sequencing technology, a total of 24,026,800 and 21,448,373 raw sequence tags were generated in the DS (P) and control (N) groups, respectively. Following removal of the low-quality reads and masking of the adaptor sequences, 21,770,279 $(\mathrm{P})$ and $17,482,100(\mathrm{~N})$ sequence tags were obtained corresponding to $280,056(\mathrm{P})$ and $232,814(\mathrm{~N})$ unique reads. Fig. 1 shows the length distribution of the small RNAs. The majority of the small RNAs from the two libraries were $\sim 22 \mathrm{nt}$ in length, which is consistent with the typical size of miRNAs generated following Dicer activity. This finding indicated that miRNAs were successively enriched from the two libraries.

The 18-30 nt sequences were then mapped to the human genome using SOAP (version 2.0), with a maximum of two mismatches. In total, 8,087,250 (P) and 12,536,630 (N) sequence tags represented by $58,523(\mathrm{P})$ and $73,833(\mathrm{~N})$ unique reads
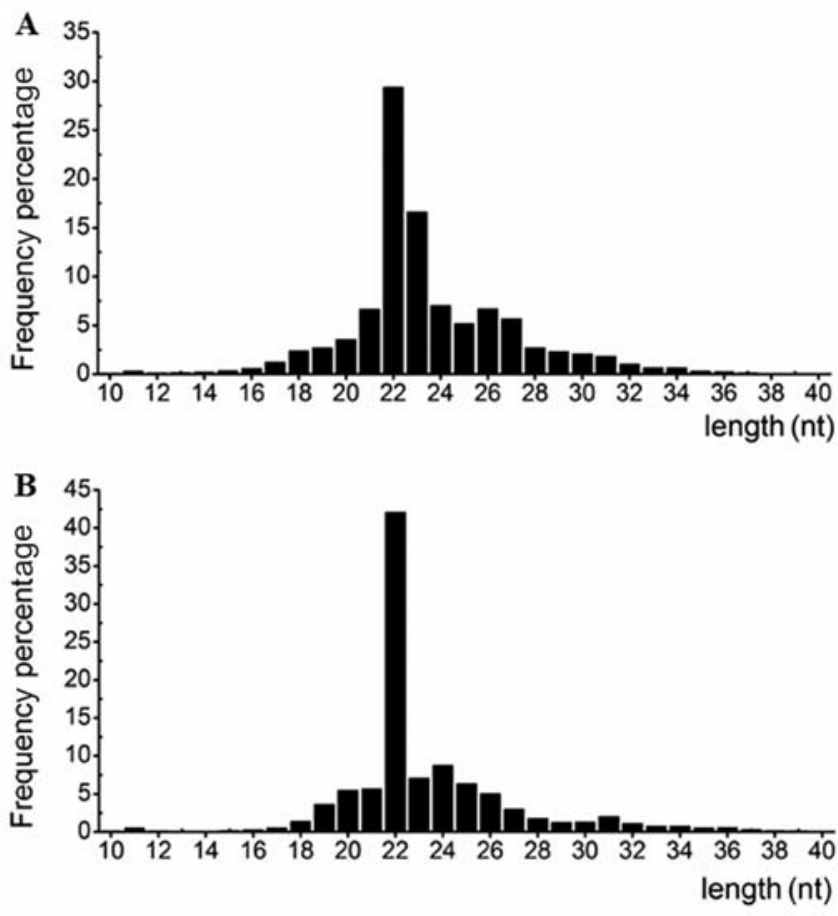

Figure 1. The nucleotide length distribution of RNA species present in the (A) control and (B) DS groups. The bar charts show the distribution of sequence tags lengths obtained from the small RNA (sRNA) libraries of the DS and control groups. The two libraries accumulated 22 nucleotide sRNAs, which is consistent with the typical size of miRNAs.

were detected to match the human genome. The sequences tags were annotated and classified into different categories (miRNA, tRNA, rRNA, mRNA, sn/snoRNA, piRNA, other non-coding RNAs and unannotated RNAs) according to their overlap with the publicly available genome annotations (data not shown). For miRNA, 2,208,096 (P) and 6,369,123 (N) sequence tags were annotated in $(\mathrm{P})$ and $(\mathrm{N})$, respectively. The surplus unannotated sequence tags served as a source of novel miRNAs.

Known miRNA expression patterns. In this study, a total of 395 miRNAs were detected from the two libraries based on miRBase 17.0. To identify the differentially regulated miRNAs in the two samples, we initially normalized each miRNA count to TPM, as described in Materials and methods. The results showed that different miRNAs exhibited significantly different expression levels ranging from $<1$ to $>10,000$ TPM (Fig. 2). Several members of the let-7 family, miR-103a, miR-107, miR-15b, miR-16, miR-185 and miR-320a were observed as some of the highly expressed miRNAs. While the members of the let-7 family, let-7a, c, d, e, f and $g$ represented $\sim 43.7 \%$ of the total miRNA counts in the (N). Notably, the let-7 family miRNAs were reported as highly abundant miRNAs in various adult tissues and are crucial in embryogenesis (26) and brain development (27).

Based on the normalized number of reads per sample, 149 miRNAs were identified as significantly differentially expressed with a fold change $>2.0$ and $\mathrm{P}<0.001$. Of the 149 significantly differentially expressed miRNAs, 143 miRNAs were downregulated and 6 miRNAs were upregulated in the DS group (Table II). In addition, another 51 miRNAs were 
Table II. The six upregulated and 14 downregulated miRNAs that were differentially expressed in the DS group (fold change $>2.0$ and $\mathrm{P}<0.001)$.

\begin{tabular}{lrrrr}
\hline & \multicolumn{2}{c}{ Relative count } & & \\
\cline { 2 - 3 } miRNA-ID & Control group & Ds group & Fold change & P-value \\
\hline hsa-miR-196b & 0.92 & 261.82 & 286.08 & 0 \\
hsa-miR-483-5p & 4.75 & 55.95 & 11.78 & $7.56 \mathrm{E}-209$ \\
hsa-miR-4732-5p & 21.85 & 241.01 & 11.03 & 0 \\
hsa-miR-320b & 61.72 & 159.71 & 2.59 & $3.11 \mathrm{E}-188$ \\
hsa-miR-486-5p & 4357.89 & 11160.35 & 2.56 & 0 \\
hsa-miR-92b* & 55.43 & 129.35 & 2.33 & $2.38 \mathrm{E}-128$ \\
hsa-miR-125b-2 & 22.88 & 8.13 & 2.81 & $3.26 \mathrm{E}-33$ \\
hsa-miR-99a & 16.93 & 4.82 & 3.51 & $1.43 \mathrm{E}-32$ \\
hsa-let-7c & 4134.17 & 986.14 & 4.19 & 0 \\
hsa-miR-16 & 3215.80 & 752.20 & 4.28 & 0 \\
hsa-miR-22 & 103.02 & 17.13 & 6.01 & $1.27 \mathrm{E}-297$ \\
hsa-miR-17 & 172.75 & 22.05 & 7.84 & 0 \\
hsa-miR-98 & 20.19 & 1.88 & 10.72 & $3.14 \mathrm{E}-79$ \\
hsa-miR-126 & 39.70 & 1.42 & 27.88 & $5.43 \mathrm{E}-198$ \\
hsa-miR-181a & 880.39 & 24.53 & 35.89 & 0 \\
hsa-miR-181b & 369.98 & 4.18 & 88.51 & 0 \\
hsa-miR-21 & 950.34 & 7.30 & 130.12 & 0 \\
hsa-miR-223 & 862.20 & 5.42 & 159.07 & 0 \\
hsa-miR-27a & 35.87 & 0.18 & 195.24 & $3.13 \mathrm{E}-212$ \\
hsa-miR-155 & 26.31 & 0.00 & $\# \mathrm{DIV} / 0 !$ & $2.30 \mathrm{E}-162$ \\
\hline
\end{tabular}

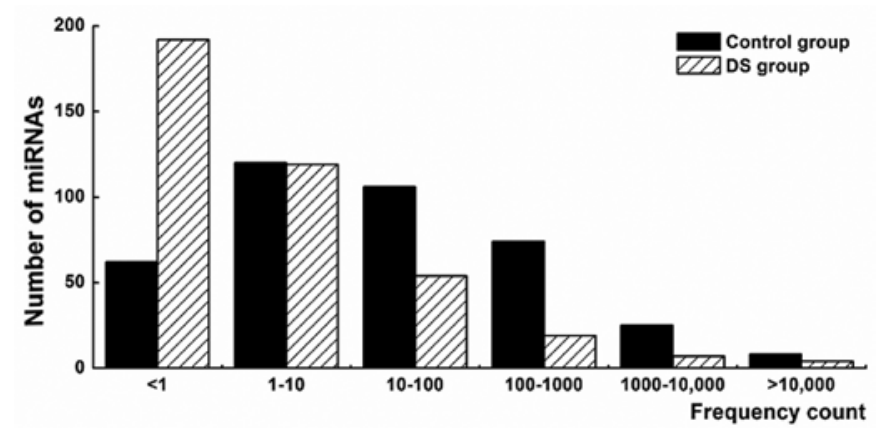

Figure 2. Overall expression levels of known miRNAs. This chart displays the distribution of the number of each known miRNA expression level. The normalized numbers of sequence tags are taken as miRNA levels and the values are represented in the form of range of values. The expression levels of miRNAs span five orders of magnitude and most of known miRNAs in the DS group are <10 TPM ( 78.7\%).

specifically expressed in the $\mathrm{N}$ group (data not shown). These specifically enriched miRNAs can be selected as candidate diagnostic biomarkers for DS fetuses. Of note, results of this experiment demonstrated that 4 of 14 Hsa21-derived miRNAs (hsa-miR-99a, let-7c, miR-125b-2 and miR-155) were downregulated in the DS group. By contrast, other Hsa21-derived miRNAs were not quantified in either of the two libraries by Illumina sequencing technology, which might be associated with the lack of primers specific for these miRNAs (3).
To validate the results of Illumina sequencing studies, we performed qPCR assays with specific stem-loop RT primers to examine the expression levels of the Hsa21-derived mature miRNAs and randomly selected 6 significantly differentially expressed miRNAs, including two upregulated miRNAs (hsamiR-196b and miR-92b*) and four downregulated miRNAs (hsa-miR-16, miR-126, miR-21 and miR-223). The results demonstrated that four Hsa21-derived mature miRNAs (hsamiR-99a, let-7c, miR-125b-2 and miR-155) were downregulated and three Hsa21-derived mature miRNAs (hsa-miR-802, miR-3648 and miR-3687) were overexpressed by at least $50 \%$ in the DS fetal CBMCs $(n=3)$ compared with matched control specimens (Fig. 3), while a further seven Hsa21-derived mature miRNAs remained undetected by stem-loop quantity PCR in all the samples. The results of six abnormally expressed non-Hsa21-derived miRNAs are shown in Fig 4. With the exception of miR-233, the changes of five abnormally expressed non-Hsa21-derived miRNAs in the DS group were consistent with the Illumina sequencing results.

Identification of novel candidate miRNAs. To identify novel candidate miRNAs from unmatched sequences in the two libraries, we first removed the annotated sequences, such as the known miRNAs, genomic repeats, coding sequences and other small RNAs. In total, 59,098 (P) and 346,626 (N) detected sequence tags were derived from unannotated regions of the human genome. As the predictions are based on identifying 


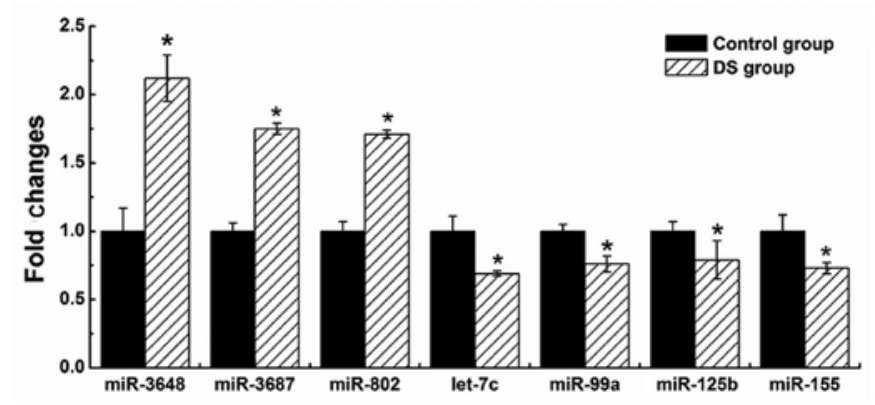

Figure 3. The expression levels of seven identified Hsa21-derived miRNAs in the DS and control specimens. Total RNA was isolated from matched DS $(n=3)$ and control $(n=3)$ fetal CBMCs with standard procedures. Of 14 mature Hsa21-derived miRNAs, only seven were detected and quantified by qPCR. Gene expression was calculated relative to RNU6B, which was assigned a value of ' 1 '. The error bars are the average \pm SE of three independent experiments using $\mathrm{n}=3$ independent specimens ( $\mathrm{P}<0.05$, DS vs. control).

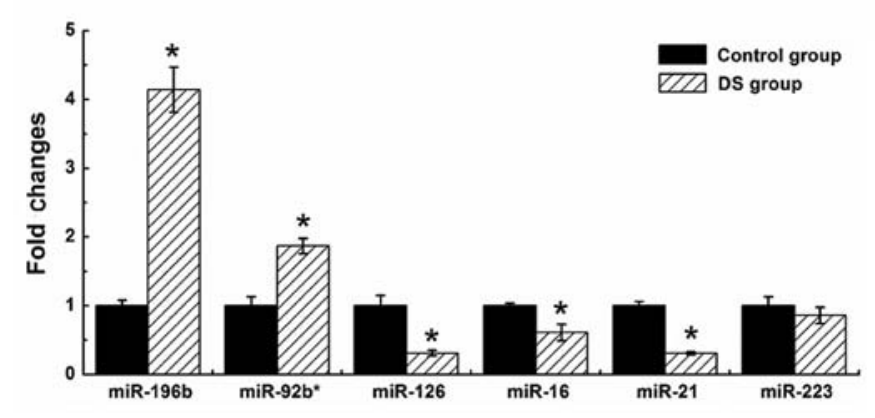

Figure 4. The expression levels of six miRNAs are validated in the DS and control specimens using qPCR. The expression of six abnormally expressed mature miRNAs was calculated relative to RNU6B, which was assigned a value of ' 1 '. The error bars are the mean $\pm \mathrm{SE}$ from three independent experiments using $\mathrm{n}=3$ independent specimens ( $\mathrm{P}<0.05$, DS vs control).
miRNA precursors, genomic regions (100 nt) surrounding these unique sequences were extracted and analyzed by MIREAP and miRDeep, which are commonly used to identify novel candidate miRNAs from high-throughput sequencing data $(19,22)$. To reduce the false-positive rate, only novel candidate miRNAs supported by these two independent tools were considered. Using this method, 181 novel candidate miRNAs were identified from the two libraries: 127 miRNAs from the $\mathrm{N}$ group and 63 miRNAs from the DS group, but 9 miRNAs were shared by both libraries.

We observed that the size of the 181 miRNAs ranged from 20 to $24 \mathrm{nt}$ and the minimum free energies of their precursors varied from -18.6 to $-71.5 \mathrm{kcal} / \mathrm{mol}$ with an average value of $-36.1 \mathrm{kcal} / \mathrm{mol}$. The length of the precursor hairpin structures ranged from 61 to $101 \mathrm{nt}$. Of note, some novel candidate miRNAs in our dataset had 1-7 isomiRs with varying frequencies, which can strengthen the prediction of these molecules as novel miRNAs (28). Additionally, two novel candidate miRNAs (designated as hsa-miR-nov-1 and hsa-miR-nov-2) genes were found to reside within the 'DS critical region' of human chromosome 21 (chr21q22.2-q22.3) (Fig. 5), in which the genes have been proven to be important in the pathologic process of DS (29). This observation suggest that the two novel miRNA genes may also be crucial in the complex and variable phenotypes of DS in the same manner as the five known Hsa21-derived miRNAs (i.e., hsa-miR-99a, let-7c, miR-125b-2, miR-155 and miR-802) (1). To validate the novel miRNAs, we performed qPCR on four randomly selected novel miRNAs (hsa-miR-nov1, hsa-miR-nov2, hsa-miR-nov3 and hsa-miRnov4), including the three aforementioned candidate miRNAs. As a result, we have successfully validated the four novel candidate miRNAs. Compared with the control samples, the

A

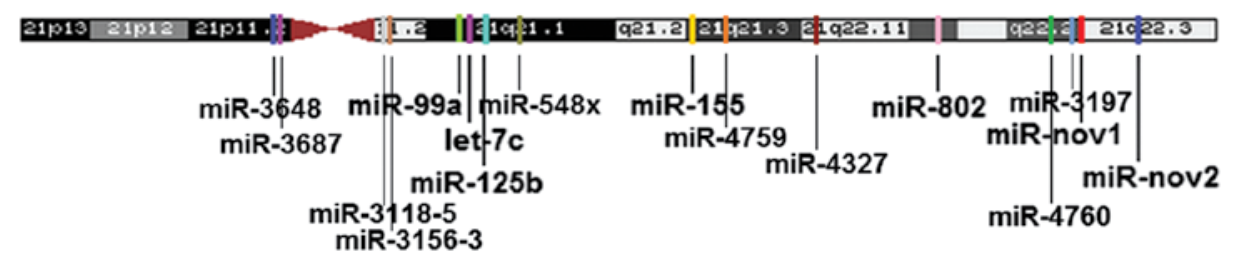

B

>hsa-niR-nov1 chr21:42580480:42580562:- 83(nt) -32.00(kcal/nol)

ATCAGAAGAATTCTGGACACGGGAGAGCACTGAAACCCTCAGAGGTAGTGTCAGCCACTCCCATGTTCAGAAGGCAGCTOCT

....... (.. $(((((((((.((((().((. .(((.(((\ldots \ldots)))) \ldots .)))))))))))))))))))).) ..) \ldots \ldots .$.

************TTCTGGACACGGGAGAGCACTG****************************************************k*k*k*k*k -TTCTGGaCACGGGaGaGGCaCTG-

Yhsa-niR-nov2 chr21:45175755:45175848:+ 94 (nt) -47.40(kcal/nol)

ACCTCACCTCTGGGAGTGGGGCCAACAGCCGGAGCOOCTCTGAGATGGGACTCAGTGCTCTCTGCCTGCOOOCCAGCOCAGCOOOCOCAACCA

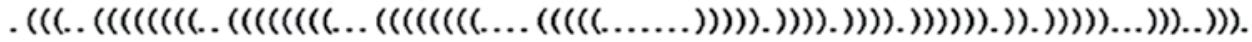

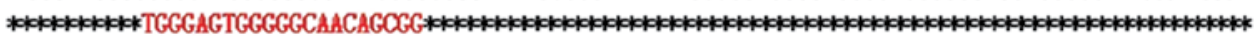
-TGGGAGTGGGGCAACAGCGG-

Figure 5. The location and precursor sequence of two Hsa21-derived novel miRNAs. (A) A map of chromosome 21 showing the locations of the 14 known and the two novel Hsa21-derived miRNAs. These data were based on the coordinates (GRCH37) available on miRBase version 17.0. The map shows that two novel candidate miRNAs reside within the 'DS critical region' (chr21q22.2-22.3) of human chromosome 21 downstream of the known 14 Hsa21-derived miRNAs. The miRNA miR-nov1 resides in the anti-sense orientation within intron 9 of the BACE2 gene and miR-nov 2 resides in the sense orientation within intron 11 of the PDXK gene. (B) The precursor sequence and the secondary structure of two Hsa21-derived novel miRNAs. The two novel candidate miRNAs together with their chromosomal locations are shown. The red letters indicate the presence of mature miRNA sequences. Notably, the sequenced mature novel candidate miRNAs map to the $5 \mathrm{p}$ arm of the precursor sequence. 


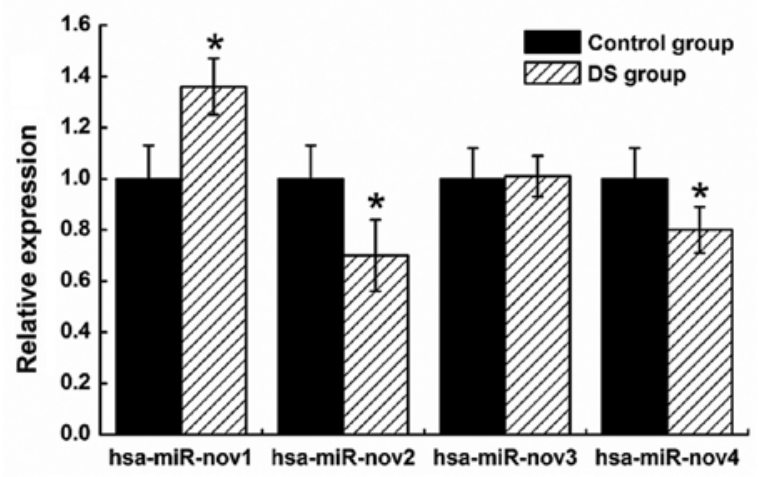

Figure 6. The expression profiles of novel mature miRNAs in the DS and control specimens using qPCR. qPCR confirms and quantifies the mature novel miRNAs that were predicted by the MIREAP and miRDeep tools. Total RNA was isolated from matched (18-22 weeks of gestation) DS ( $n=3)$ and control $(n=3)$ fetal CBMCs with standard procedures. RNU6B was used as the internal control. The error bars are the average \pm SE of three independent experiments using $\mathrm{n}=3$ independent specimens ( $(\mathrm{P}<0.05$, DS vs control).

hsa-miR-nov1 was upregulated and the hsa-miR-nov2 and miR-nov4 were downregulated in DS samples (Fig. 6).

In comparison with other miRNAs in the present study, $>92 \%$ of the putative novel miRNAs exhibited a lower expression level with $<10$ TPM after normalizing for the sequencing frequency and only 13 novel miRNAs were $>10$ TPM. This finding suggested that the majority of putative novel miRNAs may not have a significant role in DS. Nevertheless, the Hsa21-derived novel miRNAs and the novel miRNAs that measured >10 TPM may be involved in the development of the complex and variable phenotypes of DS and deserve further functional investigation.

Target prediction and GO analysis. To explore the specific functions of the differentially expressed miRNAs (fold change $>2.0$ and $\mathrm{P}<0.001$ ) in the developmental process of DS fetus, the mRNA targets of each differentially expressed miRNA were identified by miRecord tool (http://mirecords. biolead.org/) as described in Materials and methods. Five mRNA targets (i.e., SOD1, MXD4, PBX1, BCLAF1 and FOXO1) of differentially expressed miRNAs were also quantified by qPCR in the (P) and (N) (Fig. 7). By examining the GO 'biological process' classifications at level 5, results showed that the cluster of overrepresented GO terms among the predicted target genes of the up- and downregulated miRNAs in the DS fetus were involved in the regulation of transcription (GO:0045449, GO:0045941, GO:0045893), gene expression (GO:0010468, GO:0010628), cellular biosynthetic process (GO:0031326, GO:0031328), macromolecule biosynthetic process (GO:0010556, GO:0010604) and the regulation of nucleobase, nucleoside, nucleotide and nucleic acid metabolic process (GO:0019219) $(\mathrm{P}<0.001)$ (Fig. 8).

For the highly abundant $(>1,000$ TPM) and significantly differentially expressed miRNAs, with the exception of the aforementioned biological function, some clusters of significant GO terms and their mRNA targets were also associated with nervous system development, i.e., neurogenesis (GO:0022008), generation of neurons (GO:0048699), neuron differentiation and development (GO:0045664, GO:0048666), which might be associated with the pathogenesis of cognitive

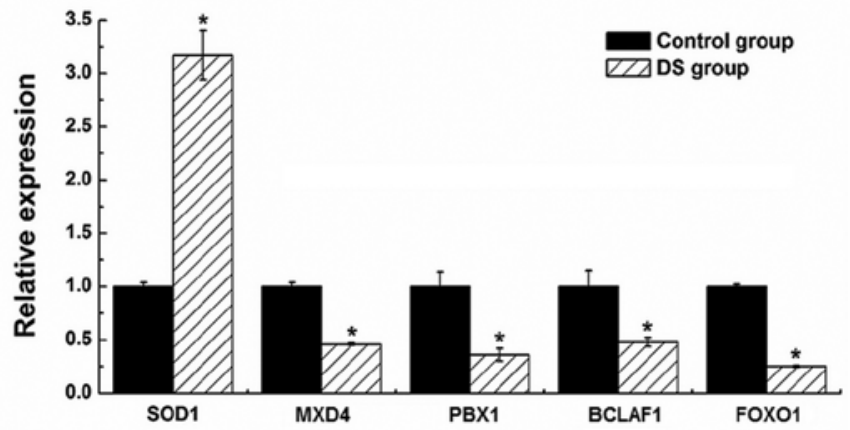

Figure 7. qPCR analysis of five mRNA targets of differentially expressed miRNAs. The expression levels of these five mRNA target genes were measured in three DS and three control fetal CBMCs using qPCR according to the instructions. Values were normalized to $18 \mathrm{~s}$ rRNA as an internal control. The error bars are the average $\pm \mathrm{SE}$ of three independent experiments using $\mathrm{n}=3$ independent specimens. ( $\mathrm{P}<0.05$, DS vs control).

impairment in DS patients. The results also suggest that a set of highly abundant and significantly differentially expressed miRNAs may promote the progression of cognitive impairment in DS patients by regulating genes in the pathway of nervous system development.

\section{Discussion}

To investigate the changes of genome-wide miRNA expression of DS fetuses under the influence of trisomy 21 during the development of DS fetus and to identify whether another miRNA gene is located on the Hsa21, we have globally examined miRNA expression profiles in the DS fetus and their normal counterparts using Illumina deep sequencing technology. In total, 395 known and 181 novel miRNAs were identified in this experiment and two novel candidate miRNAs were identified as residing within the 'DS critical region' of chromosome 21 (chr21q22.2-22.3).

Compared with the miRNAs common to the two libraries, the majority of differentially expressed miRNAs, including four Hsa21-derived miRNAs (hsa-miR-99a, let-7c, miR-125b-2 and miR-155) were downregulated in the DS group. Notably, the aberrant expression of these Hsa21derived miRNAs including has-miR-802 has been proven to contribute to the complex and variable phenotypes of DS (1). The miRNAs hsa-miR-155 and miR-802 are able to repress the expression of the $\mathrm{MeCP} 2$ gene leading to cognitive impairment in individuals with DS (4). Additionally, hsa-miR-155 has also been found to be important in the development of immunocytes during inflammation through the regulation of Bach1, Sla, Cutl1, Csflr, Jarid2, Cebp- $\beta$, PU.1, Arntl, Hifl- $\alpha$ and Picalm, while its sustained expression in the hematopoietic stem cells (HSC) is likely to lead to a myeloproliferative disorder (14). The miRNAs hsa-miR-99a, let-7c and miR-125b act as tumor suppressors and are capable of reducing the incidence of solid tumors in DS patients (16-18). However, with the exception of hsa-miR-802 being overexpressed by at least $50 \%$, the remaining four hsa21-derived miRNAs were downregulated in the DS fetal CBMCs in the present study. These results are not consistent with previous studies $(3,5)$ 


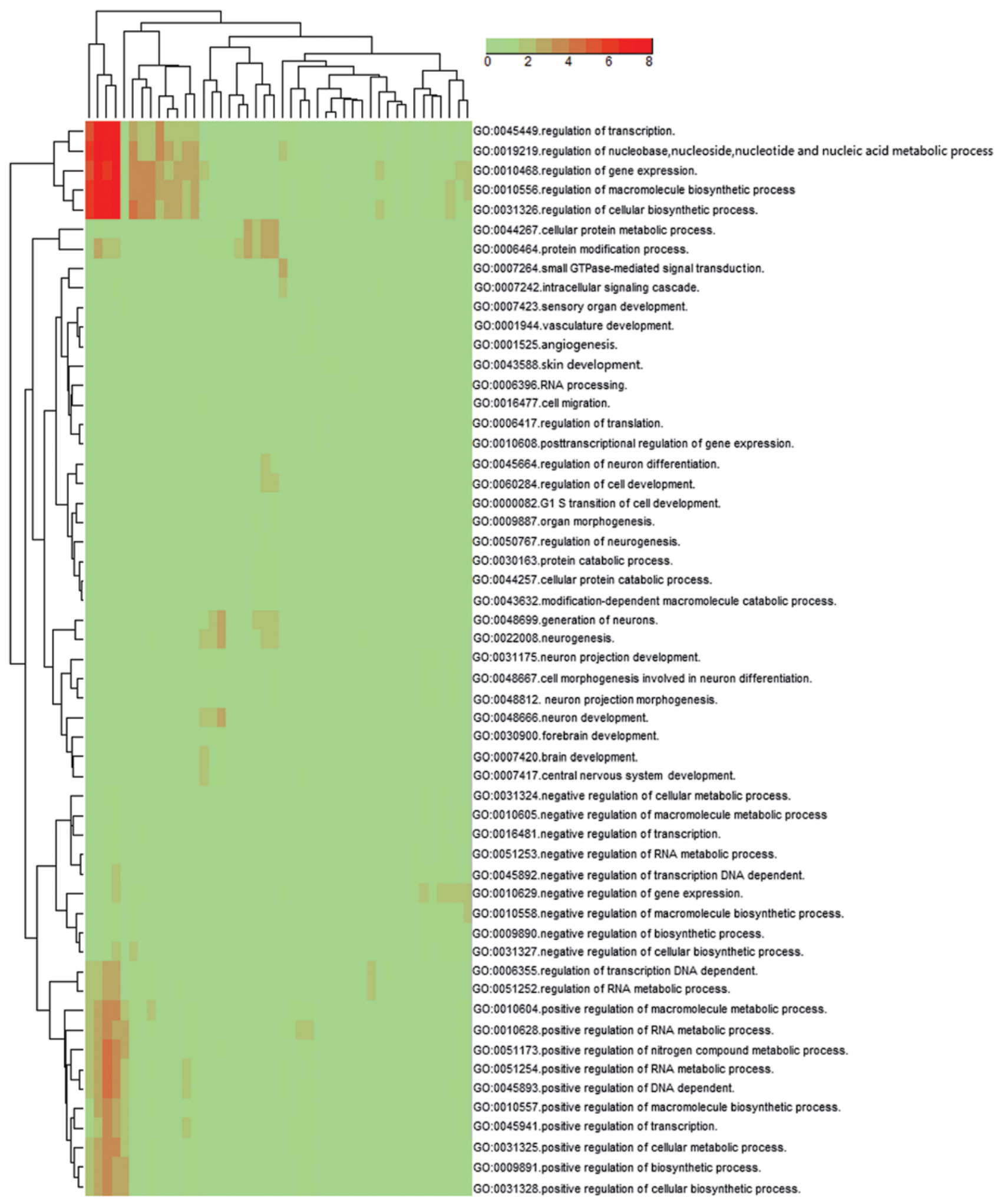

Figure 8. Clustering of overrepresented Gene Ontology (GO) classes in predicted targets of differential miRNAs (fold change $>2.0$ and $\mathrm{P}<0.001$ ). All genes with statistically overrepresented GO annotations were included $(\mathrm{P}<0.001)$.

that described these five miRNAs as being overexpressed in DS fibroblast, brain and heart cells (1). Concerning the discrepancies between the data obtained in our study and previous studies, the most important reasons may be due to the different samples (cord blood cell and fetal tissues). Two groups extensively analyzed the overexpressed Hsa21 genes in different trisomic cell/tissue types (30,31). Prandini et al (31) found that, when the lymphoblastoid cell lines were compared to the fibroblast cell lines derived from individuals with DS and euploid control individuals, only $39 \%$ of Hsa21 genes in lymphoblastoid cell lines and $62 \%$ in fibroblast cell lines were overexpressed. Li et al (30) compared the difference 
of Hsa21 gene expression between the fibroblast and fetal hearts with trisomy 21 and observed that the proportion of Hsa21 overexpressed genes in fibroblast and fetal hearts was different (29 and $15 \%$, respectively). Additionally, the discrepancies between the relative abundance of miRNA detected by Illumina sequencing technology and stem-loop qPCR may be explained by the individual variation of specimens and the application of different methods for miRNA preparation and data analysis. Nonetheless, we may preliminarily describe the miRNA expression profile of CMBC in the development of DS fetus. Additionally, the data obtained in our study should be validated using more DS cord blood samples.

Genome-wide miRNA expression profiling is likely to enhance our understanding of miRNAs and their roles in the development of the DS fetus. Numerous identified differentially regulated miRNAs in this study have been reported to be involved in various cell processes, such as cell proliferation, differentiation, apoptosis, granulocyte maturation and activation. For example, hsa-miR-233, a myeloid-specific miRNA, negatively regulates myeloid progenitor proliferation, granulocyte differentiation and activation and is involved in maintaining the function of neutrophils. In the absence of hsa-miR-233, granulocytes are hypermature and hypersensitive to activating stimuli and exhibit increased fungicidal activity (12). Moreover, hsa-miR-196b and miR-21 have been found to be involved in granulopoiesis. Their co-expression can completely block G-CSF-induced granulopoiesis (32). Additionally, the downregulation of hsa-miR-21 is able to restrain the generation of mononuclear cells and the differentiation of dendritic cells (DC), leading to the decrease of DC generation, and resulting in the phagocytic function of DC inhibition (33). DC lacking hsa-miR-155 may further impair its antigen presentation capacity, rendering it unable to induce efficient T-cell activation in response to antigens (34). Hsa-miR155 , a requirement miRNA for normal immune function, is important in the function of $\mathrm{B}$ and $\mathrm{T}$ lymphocytes and dendritic cells (35). Hsa-miR-155 deficiency results in CD4+ T cells being more prone towards Th- 2 differentiation as compared with Th-1, leading to a reduced number of germinal center B cells and extra-follicular B cells, with B cells failing to produce high-affinity IgG antibodies (36). Furthermore, some miRNAs, including hsa-miR-125a, miR-125b, miR-99b and let-7e are preferentially expressed by the actively dividing centroblasts in germinal centers and hsa-miR-125b overexpression is capable of inhibiting the differentiation of primary B cells (37).

The GO 'biological process' classifications showed that GO categories associated with the regulation of transcription, gene expression, cellular biosynthetic process, macromolecule biosynthetic process and the regulation of nucleobase, nucleoside, nucleotide and nucleic acid metabolic process were enriched among the target genes of significantly differentially expressed miRNAs in the DS fetal CBMC. The majority of mRNA targets in these categories have a well-documented association with immune modulation, including SOD1, MXD4, $P B X 1, B C L A F 1$ and $F O X O 1$. These genes were targeted by hsa-miR-1, miR-320b, miR-196b, miR-4732-5p and miR$486-5 \mathrm{p}$, respectively. The $S O D 1$ gene has been localized to the 'DS critical region' of chromosome 21 . The overexpression of SOD1 may lead to a decrease in lymphoproliferative responses, an increase in necrosis and apoptosis of T-cells in vitro and play a crucial role in T-cell functional deficits (38). MXD4, a member of the Myc-Max-Mad network, has been proven to be associated with the survival of Ag-specific T cells. The downregulation of $M X D 4$ may lead to an increase of T-cell death (39). As a major global developmental regulator, $P B X 1$ is involved in the promotion of progenitor cell proliferation in multiple tissues and has a crucial role in maintaining the postnatal hematopoietic compartment. The deletion of $P B X I$ in the thymus may lead to T- and B-lineage cell reduction (40). Bcl-2 associated factor 1 (BCLAF1) is a nuclear protein that is essential for proper homeostasis of T- and B-cell lineages and the activation-induced proliferation of $\mathrm{T}$ cells (41). Bclafl-deficient neonates exhibiting $\mathrm{T}$ cells were found to have an activation-dependent proliferation defect ex vivo (42). Furthermore, the Forkhead-box transcription factor (FOXOl) is crucial in maintaining the immune system homeostasis and was preferentially expressed in mature peripheral $\mathrm{T}$ and B cells (43). The constitutive deletion of Foxol in the T-cell lineage as well as acute deletion may lead to T-cell homing defects to peripheral lymphoid organs (44). FOXO1-deficient mature $B$ cells may fail to undergo class-switch recombination with the resulting deficiency in IgG production (45). Combined with the decrease in the number of immune cells in the blood circulatory system (46) and infection (47) presented in the DS patients, we extrapolate that the differentially expressed miRNAs might be involved in hemopoietic abnormalities and the immune defects of DS fetuses and newborns.

Of note, with the exception of the aforementioned results, target prediction of the miRNA pattern also revealed that some highly abundant and significantly differentially expressed miRNAs might be associated with the pathway of nervous system development, i.e., neurogenesis, generation of neurons, neuron differentiation and development. Notably, we found that the nervous system development genes $B D N F$ and $C D K 5 R 1$ were the targets of mir-103 family miRNAs (miR-103a/107). $B D N F$ and $C D K 5 R 1$ are important for neuron proliferation, differentiation, survival and neuronal migration $(48,49)$, suggesting that mir-103 family members might be associated with the pathogenesis of cognitive impairment in DS patients. Cognitive impairment was the common deleterious phenotype of all the DS patients. Additional investigation of the differential expression patterns of miRNAs, together with follow-up information in the clinic, may reveal a miRNA signature as a biomarker for the detection of cognitive impairment in DS patients.

Another aim of this study was to identify whether another miRNA gene is located in the human chromosome 21. Notably, 2 of 181 novel candidate miRNAs (hsa-miR-novl and hsamiR-nov2) were identified in the 'DS critical region' of human chromosome 21 (Fig. 5). The lengths of hsa-miR-nov1 and hsa-miR-nov 2 are 23 and $21 \mathrm{nt}$, respectively. The minimum free energy of both novel miRNAs is lower than $-18 \mathrm{kcal} / \mathrm{mol}$. By using the UCSC platform, we found that the hsa-miR-nov1 gene resides in the anti-sense orientation within intron 9 of the $\beta$-site APP cleaving enzyme 2 (BACE2) gene, which is located at the chr21q22.2-22.3 and plays an important role in the neurodegeneration and consequent dementia in AD and DS $(50,51)$. The hsa-miR-nov2 is located 2.6 million base pairs downstream from the hsa-miR-novl gene in the sense orientation within intron 11 of the human pyridoxal kinase 
$(P D X K)$ gene at Hsa21 genomic position q22.3. Significantly, the expression mechanism of miRNA (10) suggests that the expression of hsa-miR-nov1 and hsa-miR-nov2 might be associated with the BACE2 and $P D X K$, respectively.

To speculate on the function of these two novel candidate miRNAs, TargetScanS Custom (version 5.2) and GO were used to predict their putative targets and analyze their biological functions, respectively. The results showed that hsa-miR-nov1 has 211 conserved targets, including the $M E C P 2$ gene, which is also the target of hsa-miR-155, miR-802, let-7c and miR$125 \mathrm{~b}$ (1) and may be associated with cognitive impairment in DS individuals (4). The hsa-miR-nov2 miRNA regulates 101 conserved targets with a total of 105 conserved sites. The cluster of overrepresented GO classes in the predicted targets of the two novel miRNAs reveal that these miRNAs are mainly associated with regionalization (GO:0003002), regulation of cell differentiation (GO:0045595, GO:0045646 and GO:0045637), embryonic morphogenesis and development (GO:0048598 and GO:0009790) and ion transport (GO:0006811). However, although we have identified that hsa-miR-novl and hsa-miRnov2 were up- and downregulated in the DS fetal CBMCs (Fig. 6), respectively, the regulatory mechanisms of the two novel miRNAs have yet to be confirmed.

In conclusion, the present study has provided considerable insight into understanding the expression characteristic of miRNAs in the DS fetal CBMCs and that the differentially expressed miRNAs may be involved in hemopoietic abnormalities and the immune defects observed in DS fetuses and newborns. Additionally, certain highly abundant and differentially expressed miRNAs might be involved in the pathway of cognitive impairment of DS patients. To the best of our knowledge, this is the first study to examine genome-wide miRNA expression profiling in the DS fetus. Notably, the regulation mechanism of annotated- and novel-hsa21-derived miRNAs in the development of DS blood cells and the association between hsa21-derived miRNAs and the various phenotypes of DS should be further investigated. Specifically, the significantly differentially and specifically expressed miRNAs have the potential to be selected as biomarkers in order to construct a new antenatal diagnostic method for the DS fetus.

\section{Acknowledgements}

We are grateful to the patients for participating in this study. We thank Dr Jianhua Yang (Sun Yat-sen University, Guangzhou, P.R. China) and Wangmin Qiao (BGI, Shenzhen, P.R. China) for their helpful comments regarding the data analysis. We would also like to thank Zhaoyang Luo (Harbin Institute of Technology Shenzhen Graduate School, Shenzhen, P.R. China) for his technical assistance. This study was supported by the key project of Shenzhen S\&T program (no. 201001006) and Guangdong provincial S\&T program (no. 2012B032000008).

\section{References}

1. Elton TS, Sansom SE and Martin MM: Trisomy-21 gene dosage over-expression of miRNAs results in the haploinsufficiency of specific target proteins. RNA Biol 7: 540-547, 2010.

2. Kozomara A and Griffiths-Jones S: miRBase: integrating microRNA annotation and deep-sequencing data. Nucleic Acids Res 39: D152-157, 2011.
3. Kuhn DE, Nuovo GJ, Martin MM, et al: Human chromosome 21-derived miRNAs are overexpressed in down syndrome brains and hearts. Biochem Biophys Res Commun 370: 473-477, 2008.

4. Kuhn DE, Nuovo GJ, Terry AV, Jr, et al: Chromosome 21-derived microRNAs provide an etiological basis for aberrant protein expression in human Down syndrome brains. J Biol Chem 285: 1529-1543, 2010.

5. Sethupathy P, Borel C, Gagnebin M, et al: Human microRNA-155 on chromosome 21 differentially interacts with its polymorphic target in the AGTR1 3' untranslated region: a mechanism for functional single-nucleotide polymorphisms related to phenotypes. Am J Hum Genet 81: 405-413, 2007.

6. Lewis BP, Burge CB and Bartel DP: Conserved seed pairing, often flanked by adenosines, indicates that thousands of human genes are microRNA targets. Cell 120: 15-20, 2005.

7. Cheng AM, Byrom MW, Shelton J and Ford LP: Antisense inhibition of human miRNAs and indications for an involvement of miRNA in cell growth and apoptosis. Nucleic Acids Res 33: 1290-1297, 2005

8. Chen CZ, Li L, Lodish HF and Bartel DP: MicroRNAs modulate hematopoietic lineage differentiation. Science 303: 83-86, 2004.

9. Vaz C, Ahmad HM, Sharma P, et al: Analysis of microRNA transcriptome by deep sequencing of small RNA libraries of peripheral blood. BMC Genomics 11: 288, 2010.

10. Bartel DP: MicroRNAs: genomics, biogenesis, mechanism, and function. Cell 116: 281-297, 2004.

11. Bandiera S, Hatem E, Lyonnet S and Henrion-Caude A: microRNAs in diseases: from candidate to modifier genes. Clin Genet 77: 306-313, 2010.

12. Havelange V and Garzon R: MicroRNAs: emerging key regulators of hematopoiesis. Am J Hematol 85: 935-942, 2010.

13. Szulwach KE, Jin P and Alisch RS: Noncoding RNAs in mental retardation. Clin Genet 75: 209-219, 2009.

14. O'Connell RM, Rao DS, Chaudhuri AA, et al: Sustained expression of microRNA-155 in hematopoietic stem cells causes a myeloproliferative disorder. J Exp Med 205: 585-594, 2008.

15. Malinge S, Izraeli S and Crispino JD: Insights into the manifestations, outcomes, and mechanisms of leukemogenesis in Down syndrome. Blood 113: 2619-2628, 2009.

16. Ozen M, Creighton CJ, Ozdemir M and Ittmann M: Widespread deregulation of microRNA expression in human prostate cancer. Oncogene 27: 1788-1793, 2008.

17. Nagayama K, Kohno T, Sato M, Arai Y, Minna JD and Yokota J: Homozygous deletion scanning of the lung cancer genome at a 100-kb resolution. Genes Chromosomes Cancer 46: 1000-1010, 2007.

18. Calin GA, Sevignani C, Dumitru CD, et al: Human microRNA genes are frequently located at fragile sites and genomic regions involved in cancers. Proc Natl Acad Sci U S A 101: 2999-3004, 2004.

19. Creighton CJ, Reid JG and Gunaratne PH: Expression profiling of microRNAs by deep sequencing. Brief Bioinform 10: 490-497, 2009.

20. Li R, Yu C, Li Y, et al: SOAP2: an improved ultrafast tool for short read alignment. Bioinformatics 25: 1966-1967, 2009.

21. Anders S and Huber W: Differential expression analysis for sequence count data. Genome Biol 11: R106, 2010.

22. Xu G, Wu J, Zhou L, et al: Characterization of the small RNA transcriptomes of androgen dependent and independent prostate cancer cell line by deep sequencing. PLoS One 5: e15519, 2010.

23. Chen $\mathrm{X}, \mathrm{Li} \mathrm{Q}$, Wang $\mathrm{J}$, et al: Identification and characterization of novel amphioxus microRNAs by Solexa sequencing. Genome Biol 10: R78, 2009.

24. An J, Lai J, Lehman ML and Nelson CC: miRDeep*: an integrated application tool for miRNA identification from RNA sequencing data. Nucleic Acids Res 41: 727-737, 2013.

25. Castillo-Davis CI and Hartl DL: GeneMerge-post-genomic analysis, data mining, and hypothesis testing. Bioinformatics 19: 891-892, 2003.

26. Schulman BR, Esquela-Kerscher A and Slack FJ: Reciprocal expression of lin-41 and the microRNAs let-7 and mir-125 during mouse embryogenesis. Dev Dyn 234: 1046-1054, 2005.

27. Wulczyn FG, Smirnova L, Rybak A, et al: Post-transcriptional regulation of the let-7 microRNA during neural cell specification. FASEB J 21: 415-426, 2007.

28. Morin RD, O'Connor MD, Griffith M, et al: Application of massively parallel sequencing to microRNA profiling and discovery in human embryonic stem cells. Genome Res 18: 610-621, 2008.

29. Patterson D: Molecular genetic analysis of Down syndrome. Hum Genet 126: 195-214, 2009. 
30. Li CM, Guo M, Salas M, et al: Cell type-specific over-expression of chromosome 21 genes in fibroblasts and fetal hearts with trisomy 21. BMC Med Genet 7: 24, 2006.

31. Prandini P, Deutsch S, Lyle R, et al: Natural gene-expression variation in Down syndrome modulates the outcome of genedosage imbalance. Am J Hum Genet 81: 252-263, 2007.

32. Velu CS, Baktula AM and Grimes HL: Gfil regulates miR-21 and miR-196b to control myelopoiesis. Blood 113: 4720-4728, 2009.

33. Gracias DT and Katsikis PD: MicroRNAs: key components of immune regulation. Adv Exp Med Biol 780: 15-26, 2011.

34. Lu LF and Liston A: MicroRNA in the immune system, microRNA as an immune system. Immunology 127: 291-298, 2009.

35. Rodriguez A, Vigorito E, Clare S, et al: Requirement of bic/ microRNA-155 for normal immune function. Science 316 : 608-611, 2007.

36. Fernando TR, Rodriguez-Malave NI and Rao DS: MicroRNAs in B cell development and malignancy. J Hematol Oncol 5: 7, 2012.

37. Gururajan M, Haga CL, Das S, et al: MicroRNA 125b inhibition of B cell differentiation in germinal centers. Int Immunol 22: 583-592, 2010

38. Banerjee R, Mosley RL, Reynolds AD, et al: Adaptive immune neuroprotection in G93A-SOD1 amyotrophic lateral sclerosis mice. PLoS One 3: e2740, 2008.

39. Vasilevsky NA, Ruby CE, Hurlin PJ and Weinberg AD: OX40 engagement stabilizes Mxd4 and Mnt protein levels in antigenstimulated $\mathrm{T}$ cells leading to an increase in cell survival. Eur J Immunol 41: 1024-1034, 2011.

40. Ficara F, Murphy MJ,Lin M and Cleary ML: Pbx1 regulates selfrenewal of long-term hematopoietic stem cells by maintaining their quiescence. Cell Stem Cell 2: 484-496, 2008.

41. McPherson JP, Sarras H, Lemmers B, et al: Essential role for Bclaf1 in lung development and immune system function. Cell Death Differ 16: 331-339, 2009.
42. Sarras H, Alizadeh Azami S and McPherson JP: In search of a function for BCLAF1. ScientificWorldJournal 10: 1450-1461, 2010.

43. Dejean AS, Hedrick SM and Kerdiles YM: Highly specialized role of Forkhead box $\mathrm{O}$ transcription factors in the immune system. Antioxid Redox Signal 14: 663-674, 2011.

44. Ouyang W, Beckett O, Flavell RA and Li MO: An essential role of the Forkhead-box transcription factor Foxol in control of T cell homeostasis and tolerance. Immunity 30: 358-371, 2009.

45. Dengler HS, Baracho GV, Omori SA, et al: Distinct functions for the transcription factor Foxol at various stages of B cell differentiation. Nat Immunol 9: 1388-1398, 2008.

46. de Hingh YC, van der Vossen PW, Gemen EF, et al: Intrinsic abnormalities of lymphocyte counts in children with Down syndrome. J Pediat 147: 744-747, 2005.

47. Ram $\mathrm{G}$ and Chinen J: Infections and immunodeficiency in Down syndrome. Clin Exp Immunol 164: 9-16, 2011.

48. Lee J, Duan W and Mattson MP: Evidence that brain-derived neurotrophic factor is required for basal neurogenesis and mediates, in part, the enhancement of neurogenesis by dietary restriction in the hippocampus of adult mice. J Neurochem 82: 1367-1375, 2002.

49. Zuccotti P, Barbieri A, Colombrita C, et al: Identification of post-transcriptional regulatory elements in CDK5R1 3'UTR gene involved in CNS development and functioning. Eur J Hum Genet 19: 354, 2011.

50. Holler CJ, Webb RL, Laux AL, et al: BACE2 expression increases in human neurodegenerative disease. Am J Pathol 180: 337-350, 2012.

51. Webb RL and Murphy MP: $\beta$-Secretases, Alzheimer's disease, and Down syndrome. Curr Gerontol Geriatr Res 2012: 362839, 2012. 